\title{
The perception of clicks in linguistic and nonlinguistic messages ${ }^{1}$
}

\author{
ARTHUR S. REBER ${ }^{2}$ AND JOHN R. ANDERSON ${ }^{3}$ \\ UNIVERSITY OF BRITISH COLUMBIA
}

\begin{abstract}
Three experiments were run analyzing Ss' abilities to locate clicks in auditory messages. In Experiment 1 it was found that the intonation pattern of the linguistic message largely determined the types of emors $S s$ made in click placement; syntactic factors were only marginally effective and semantic factors were effectively irrelevant. In addition to these linguistic factors, there was evidence that such nonlinguistic factors as attention, memory, and response biases were contributing to the data. Experiment 2 supplied additional evidence favoring these nonlinguistic factors. Experiment 3 was a mock-up of Experiment 2 except that broad-band white noise was used as the primary message instead of English sentences. The patterns of placement errors Ss made in these several experiments (especially Experiments 2 and 3) were sufficiently similar to one another to force the conclusion that nonlinguistic factors are primarily responsible for the errors $S s$ make in trying to locate clicks in messages. A neo-Titchenerian attention hypothesis based upon the law of prior entry was proposed to account for the data.
\end{abstract}

In a widely referenced article, Fodor and Bever (1965) argued that constituent structure "plays an important role in determining the subjective location of noise perceived during speech." As evidence to support this conclusion they cited certain characteristics of the distribution of errors Ss made in attempting to locate the position of a short click sound occurring in sentential material. Specifically, they found that the boundaries of constituents acted as perceptual as well as linguistic boundaries. Thus, they argued that it is reasonable to regard speech perception as an "active" process where the hearer segments the input on the basis of an abstract syntactic analysis based upon constituent structure.

We do not quarrel with this interpretation of speech perception for it is supported by a great deal of data collected with a wide variety of experimental paradigms (cf. Neisser, 1967; Miller \& Isard, 1963; Marks \& Miller, 1964; among others). We do, however, question their statement that their data clearly substantiate it. There were a number of procedural problems with their experiment and with their data analyses that make their interpretation suspect.

It seems premature to regard a constituent structure analysis of a grammatical sentence as revealing the psychological and linguistic segments by which the sentence is perceived. Clearly, the analysis of any linguistic input must involve a complex set of operations. As a beginning, we feel that it is essential to distinguish between the linguistic and the nonlinguistic operations. By linguistic, we refer to operations based upon such factors as intonation, syntax, and semantics. By nonlinguistic, we refer to operations based upon such factors as memory and attention. We do not feel that this dichotomization is either inclusive or exhaustive. These two operative levels undoubtedly interact, and probably in subtle ways, but we shall not discover the ways unless we perform comprehensive experiments.

The experiments reported below were run in an effort to separate empirically the linguistic and nonlinguistic operations that Ss use in the perception of a short burst of interfering noise (clicks) in an auditory message. The first two studies represent an effort to evaluate systematically the effects of the linguistic factors of intonation, syntax, and semantics in determining $\mathrm{Ss}^{\circ}$ perception of the interfering stimulus. The third study represents an effort to isolate the role of nonlinguistic memory and attention processes as they operate within a similar setting.

The experimental procedure in all cases involved the perception of extraneous noise superimposed upon an auditory message. We implicitly assume (with Fodor and Bever) that the pattern of errors that Ss make in attempting to locate the position of the noise relative to the primary message contains information about the manner in which the inputs are being processed.

\section{EXPERIMENT 1}

The paradigm for this experiment was adapted from that of Fodor and Bever (1965). Certain aspects of their study shall be noted since they were modified for present purposes. First, Fodor and Bever used sentence length ranging from 8 to 22 words. Although it is impressive that such a consistent effect was obtained despite the wide range, this variation makes it impossible to manipulate sentence structure in a systematic way. In this experiment, stimulus length was kept constant. Second, Fodor and Bever had their Ss write down the sentences prior to marking the click position. A number of transcription errors resulted and a portion of the data had to be discarded. Moreover, with this procedure, it is impossible to control either (1) the interval between hearing the sentence and marking the click position or (2) the interval between hearing the click and marking its position. It is, of course, impossible to control both intervals simultaneously if click position is to vary, but it is desirable to control one of them. In the present study, Ss were not required to transcribe the sentences, and thus it was possible to control the interval between hearing the sentence and marking the perceived click position. Finally, in the Fodor and Bever study, the primary message was presented to one ear through one earphone and the click to the other ear through another earphone. When the sentence was in the left ear, Ss tended to locate the click to the right of (i.e., later than) its actual position, but with the headphones reversed, they tended to locate the click to the left. There is no reason to assume that either condition reflected the normal processing of language. The results from the two conditions were pooled, but the sum of two biased sets of data cannot be regarded as unbiased. We presented the materials over conventional speakers.

Fodor and Bever also had no notion of the underlying response distribution. It is entirely possible that when Ss do not know the actual location of a click they have nonuniform guessing probabilities. Ladefoged (1967) reported a tendency for Ss to locate imperceptible clicks nearer the major syntactic boundaries. His experiment was a rather informal one but the results were clear enough for him to conclude, "... part of the responses recorded in ours and Fodor and Bever's experiments may have nothing to do with a subject's perception from the point of view of the way he scans incoming auditory data." Therefore, we ran a control group where Ss responded to "subliminal" but actually nonexistent clicks. The distribution of responses made by these Ss was used as a check on Ss' response biases. 
Method

Materials. This experiment independently altered the intonational, syntactic, and semantic integrity of sentences. The method of manipulating the syntactic and semantic components is that first used by Miller and Isard (1963). Intonational cues were varied by using either normal intonation or a paced monotone. This experimental design made it possible to determine which aspects of the data could be attributed to the various linguistic components of the structure that exist in speech.

The term "word string" refers to any of the six-word sequences used in this experiment. One set of 18 word strings were English sentences with similar grammatical structure (two modifiers, a plural noun subject, a present-tense transitive verb, a modifier, and a plural noun object). Each word contained two syllables. From this set of sentences, 18 additional word strings were constructed by taking the first word from one sentence, the second from another, and so on. Each word kept the same ordinal position that it had in the original sentence, but no word string contained more than one word from the same sentence. These word strings had grammatical structure but were semantically anomalous. Two more sets of 18 word strings were constructed by randomizing the order of words within the word strings of the original two sets. Thus, syntactic structure was disrupted independently of the semantic component. This process yielded four sets of 18 word strings, each set representing a combination of a syntactic factor with two levels, grammatical (G) or random (R), and a semantic factor with two levels, sentence (S) or anomalous (A). An example of each follows:

Naughty raucous children disrupt birthday parties-grammatical, sentence (GS)

Parties raucous disrupt children naughty. birthday-random, sentence (RS)

Naughty soda autos suppress flower ailments-grammatical, anomalous (GA)

Ailments soda suppress autos naughty

flower-random, anomalous (RA)

Each set of 18 was divided randomly into two equal subsets. One subset was read with normal intonation (N) (normal for English sentences like the GS word strings), while the other received a paced monotone intonation (M). Thus, an intonational factor was combined independently with the other two factors. In duration, the $\mathbf{N}$ word strings were about $3 \mathrm{sec}$ and the $M$ word strings about $5.5 \mathrm{sec}$.

The final factor, click position (C), had nine levels. These nine click placements are
Table 1

$\begin{array}{ccccccc}\text { O-pen } & \text { road-side } & \text { mar-kets } & \text { dis-play } & \text { tas-ty } & \text { pro-ducts } \\ -7 & -4 & -2-1 & 0+1+2 & +4 & +7\end{array}$

illustrated in Table 1. The central word Results space is referred to as Position 0. Each space or syllable is counted as one unit (negatively to the left of Position 0 and positively to the right). As illustrated, one click was placed over Position 0 and eight others symmetrically about this position. One word string from each subset was assigned randomly to each click position. The clicks were produced by introducing a .05 -msec burst of broad-band white noise and were set approximately equal to the most intense speech sounds.

The procedure for generating word strings yielded 72 stimulus items, each representing a different independent intonation, semantic, and syntactic factor levels in a 9 by 2 by 2 by 2 factorial design. The assignment of particular word strings to the intonation and click position factor levels was determined randomly. Two such randomizations were carried out producing two separate assignments. Order of word strings within each assignment was also randomized. The two assignments were read on to one track of a tape and the clicks were appropriately placed on the second track. The subliminal control Ss heard the same tape as the experimental Ss except that the channel containing the clicks was disconnected. The experimental Ss heard both assignments in counterbalanced order; the subliminal Ss heard only one assignment.

Procedure. Ss were tested in a quiet room free from distraction. All Ss heard three practice word strings before the actual experiment began. Test booklets were constructed that contained 72 unmarked word strings printed in capital letters without punctuation. Ss under both the click and subliminal conditions turned to the appropriate word string in their booklet only after they heard it. They marked the position of the click with an arrow and waited to hear the next word string. The subliminal Ss, of course, could not hear the clicks. They were told that there was a click, but that they would not "even be aware of hearing it" and that "experiments have shown that subjects under these conditions locate the clicks with better than chance accuracy." They were asked to mark the position they thought the "click most likely occurred."

Subjects. The Ss were 82 undergraduates at the University of British Columbia. Seventy Ss were in the experimental condition and 12 were run under the subliminal control condition.
A total of 10,080 responses were made by the $S s$ in the experimental group. Of these, 4,223 , or .419 , were errors. Of these error responses, 2,158 , or .511 , were errors to the right of the actual click position. These two measures of the Ss' responses, the probability of an error $\left(\mathrm{P}_{\mathrm{e}}\right)$ and the probability of an error to the right of the actual click position $\left(\mathrm{P}_{\mathrm{e}, \mathrm{r}}\right)$ made up the primary tools for the analyses.

Probability of an error, $P_{e}$. This measure gives an assessment of the accuracy with which Ss were able to locate clicks. Figure 1 shows how accuracy in click location is dependent upon (a) position of
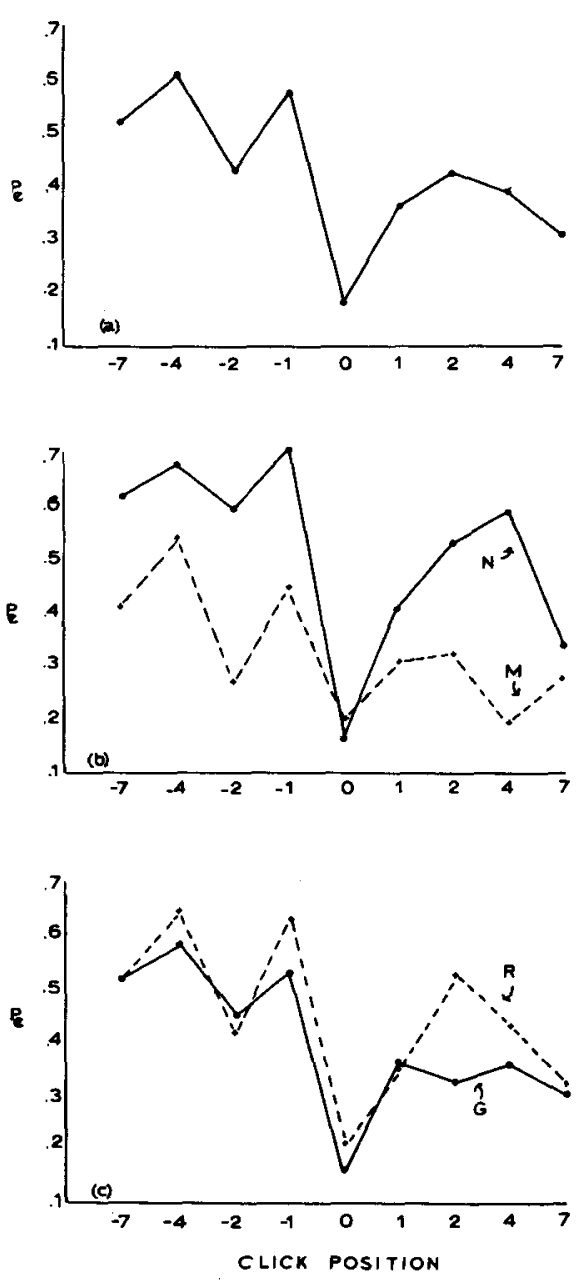

Fig. 1. Measures of accuracy: Proportion of errors $\left(P_{e}\right)$ in Experiment 1 for (a) click positions, all conditions pooled, (b) intonation pattern by click positions, and (c) syntax by click positions. 
the click, (b) the intonation pattern of the stimulus item, and (c) the syntactic structure of the stimulus item.

(a) Click position. There was a highly significant effect of click position on $\mathrm{P}_{\mathrm{e}}$, $F(8,9977)=94.22, p<.001$. The effect was contained in an overall decrease in $P_{e}$ with successive click positions and in the overall low $P_{e}$ found in Position 0 . The linear decrease in $P_{e}$ with progress through stimulus items was significant $(p<.001)$ and is presumably due to a short-term memory process. Those clicks occurring at the end of an item have a small interval between the S's hearing of the click and his marking of his response and they have less stimulus material intervening that could act as interference. Kaplan ${ }^{4}$ has run a similar study but where the Ss made their responses as soon as they heard the click rather than waiting until the end of the item. This condition produced lower $\mathbf{P}_{\mathrm{e}}$ than a control condition that was comparable to our procedure.

The extremely low $P_{e}$ found in Position 0 may be due partly to the presence of a pause in the acoustic stimulus at this point. A spectrographic analysis of each item used in the experiment revealed a number of drops in acoustic energy at Position 0, particularly in normally intonated items. Although such pauses were found in other parts of items, they occurred with more regularity here. The effect of a pause, however, is not unambiguous. A pause could allow $\mathrm{Ss}$ to locate clicks that actually occurred there more accurately, but it could also produce a disproportionately high guessing rate. That is, Ss would have more time to think they heard the click there. A disproportionately high number of responses in any one position will produce an artificially low $\mathbf{P}_{\mathbf{e}}$ there.

The data from the subliminal control group discussed below (see Fig. 3), do, in fact, reveal a strong response bias favoring central positions, particularly Position 0 . To partially correct for the effect of this response bias, we calculated a weighted $P_{e}$, denoted $P_{e}^{\prime}$. $P_{e}^{\prime}$ is an average based upon the number of errors per response rather than the number of errors per click occurrence. Thus, $\mathrm{P}_{\mathrm{e}}^{\prime}$ is given by 1 minus the ratio of the number of correct responses in a particular position to the total number of responses made in that position. The average $\mathrm{P}_{e}^{\prime} \mathrm{s}$ for the position left-of-0, 0 , and those right-of-0 were .509 , .418 , and .405 , respectively. Although this correction is only an approximation, because we do not have a theoretical underlying response distribution, it does seem fairly clear that Position 0 clicks are not necessarily easier to detect than those outside of Position 0. Note, however, that the overall decrease in $P_{e}$ with late click positions is still found after the correction and is not due to response biases.

(b) Intonation. Analysis of variance showed a highly significant effect of the pattern of intonation, $F(1,9977)=400.8$, $\mathrm{p}<.001$. The normal intonation pattern (N) produced considerably more errors than the monotone (M), a result that was not unexpected since $N$ items were only about $3 \mathrm{sec}$ in duration while $M$ items were about 5.5 sec. The value of $P_{e}$ was higher for $\mathbf{N}$ items in all click positions except Position 0. Again, we conclude that memory factors plus a response bias are responsible for these results.

(c) Syntax. The syntactic factor was also significant, $F(1,9977)=25.9, \quad p<.001$, although the effect was somewhat less dramatic than those of click position and intonation. Figure lc shows the values of $P_{e}$ for the grammatical items (G) and the random word strings (R) for each click position. Overall, the $G$ items produced fewer errors than did the $R$ items. This result is most surprising in light of interpretations of previous research. According to the linguistic decoding interpretation, the most salient factor controlling the errors Ss make in locating clicks is the syntactic structure of the iterns. The fact that our Ss made fewer errors when the items were grammatical calls this interpretation into question. Perhaps $G$ items produce fewer errors because they are easier to process and thus place less stress on the Ss' memory capacities.

Probability of an error to the right, $P_{e, r}$. This measure treats only trials on which $S$ made an error in the location of the click. The responses were scored on the basis of the direction of the error and are presented as the proportion of total errors made to the right (toward the end of the sentence) of the actual click position. Figure 2 shows the relationship between $P_{e, r}$ and (a) click position, (b) intonation, and (c) syntax.

(a) Click position. Note first that there was a slight overall bias; .511 of the total errors were to the right, and five of the nine click positions displayed a right-bias. There was a highly significant shift from a right-bias for clicks occurring early in an item to a left-bias for clicks toward the end, $F(8,9977)=70.6, p<.001$. Thus, Ss generally perceived clicks occurring at the beginning of the items as occurring later than they actually did and clicks occurring toward the end of items as occurring earlier than they actually did. Further, the tendency to locate Position -1 clicks to the right is greater than the tendency to move Position +1 clicks to the left, and fully $65 \%$ of the errors in Position 0 are to the right. This asymmetry is of particular
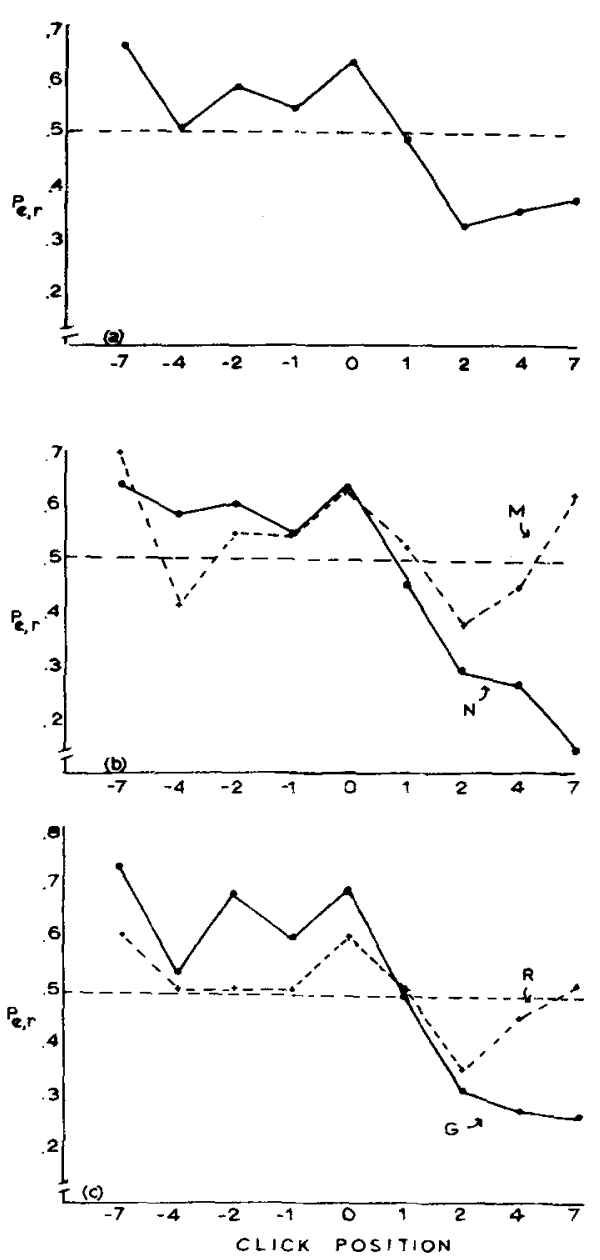

Fig. 2. Measures of directional bias: Proportion of errors to the right of actual click position $\left(P_{e, r}\right)$ in Experiment 1 for (a) click positions, all conditions pooled, (b) intonation pattern by click position, and (c) syntax by click position.

interest since the constituent structure model views the major syntactic break as "attracting" clicks from both sides symmetrically.

There was also a tendency for the direction of the error to shift over the course of the experiment. In the first block of 12 trials, average $P_{e, r}$ was only .38 ; it increased to $.40, .50$, and .53 on successive blocks of trials. The remaining trial blocks were fairly stable, with a mean of .53. This shift from a left- to a right-bias has been found in other studies (Ladefoged \& Broadbent, 1960; Fodor \& Bever, 1965).

(b) Intonation. This factor was highly significant, $F(1,9977)=80.4, p<.001$, as was the Intonation by Click Position interaction, $F(8,9977)=26.6, p<.001$. The interaction shown in Fig. $2 b$ reveals quite clearly that the sharp decrease in right-bias with late click position is due 
Table 2

Proportion of Errors "Supporting" the Linguistic Decoding Hypothesis of Fodor and Bever (1965). - indicates data averaged over that condition.

\begin{tabular}{|c|c|c|c|}
\hline Condition & $\begin{array}{c}\text { Total } \\
\text { Supporting } \\
\text { Errors }\end{array}$ & $\begin{array}{c}\text { Total } \\
\text { Number } \\
\text { of Errors }\end{array}$ & $\begin{array}{c}\text { Propor- } \\
\text { tion }\end{array}$ \\
\hline $\begin{array}{l}\text { NGS } \\
\text { NGA } \\
\text { NRS } \\
\text { NRA } \\
\text { MGS } \\
\text { MGA } \\
\text { MRS } \\
\text { MRA }\end{array}$ & $\begin{array}{l}418 \\
429 \\
388 \\
357 \\
181 \\
182 \\
226 \\
196\end{array}$ & $\begin{array}{l}612 \\
619 \\
619 \\
634 \\
366 \\
309 \\
427 \\
446\end{array}$ & $\begin{array}{l}.683 \\
.693 \\
.627 \\
.563 \\
.494 \\
.589 \\
.529 \\
.439\end{array}$ \\
\hline $\begin{array}{l}\mathrm{N}-- \\
\mathrm{M}--\end{array}$ & $\begin{array}{r}1592 \\
785\end{array}$ & $\begin{array}{l}2484 \\
1548\end{array}$ & $\begin{array}{l}.641 \\
.507\end{array}$ \\
\hline $\begin{array}{l}-\mathrm{G}- \\
-\mathrm{R}-\end{array}$ & $\begin{array}{l}1210 \\
1167\end{array}$ & $\begin{array}{l}1906 \\
2126\end{array}$ & $\begin{array}{l}.635 \\
.549\end{array}$ \\
\hline $\begin{array}{l}--S \\
--A \\
---\end{array}$ & $\begin{array}{l}1213 \\
1164 \\
2377\end{array}$ & $\begin{array}{l}2024 \\
2008 \\
4032\end{array}$ & $\begin{array}{l}.599 \\
.580 \\
.590\end{array}$ \\
\hline
\end{tabular}

primarily to the intonation pattern. Linear regression analysis showed that normally intonated items $(N)$ produced a dramatic shift in directional bias $(p<.001)$, while the monotone (M) items had comparatively little effect $(p<.10)$. However, both conditions show the right-bias at Position 0. Clearly one of the primary factors causing Ss to perceive early clicks differently than later ones is the intonation pattern of the item.

(c) Syntax. Analysis of variance failed to show any overall effect of syntactic structure on direction of error, $F<1.0$. There was, however, a significant Syntax by Click Position interaction, $F(8,9977)=9.79, \quad p<.001$, which is shown in Fig. 2c. Grammatical (G) items tended to contribute more to the shift from a right- to a left-bias than random (R) ones. Again, Position 0 produced a right-bias in both cases.

In support of their linguistic decoding hypothesis, Fodor and Bever (1965) reported that $66 \%$ of the errors were either toward or into the major syntactic break. The last line of Table 2 shows that the data from this experiment, analyzed in this fashion, reveal a similar proportion of .59 . However, as the other cells in Table 2 show, this "support" is quite complex. Note that the intonation factor was the most powerful in producing agreement with the Fodor and Bever position. With normal intonation, $64.1 \%$ of the errors are toward the major syntactic break, but with the monotone intonation only $50.7 \%$, or

Fig. 3. Distribution of placement responses made under the "subliminal" condition, normal (N) and monotone (M) intonation patterns plotted separately. roughly chance, were toward the major break. In fact, the MGS items that are both meaningful and grammatical produced only 49.4\% supportive errors. Further, the syntax factor, which should be critical in controlling the direction of errors, was less effective than intonation, with $63.5 \%$ for grammatical items and $54.9 \%$ for random items. Note that this $54.9 \%$ occurs even though the major syntactic break does not exist in the nongrammatical $R$ items.

The "subliminal" condition. The signifcant effects were those produced by click position, $\chi^{2}(16)=126.8, p<.001$, and by the Click Position by Intonation interaction, $\chi^{2}(16)=45.6, p<.001$. Figure 3 gives the total number of placements in each position in the $N$ and $M$ intonation conditions. There is a strong central tendency in both conditions, although it is much more pronounced under $\mathrm{N}$. The data are quite clear in showing that the underlying distribution of Ss' responses is nonuniform with the center of an item attracting a dominant proportion of the responses. As we argue later, this nonuniform distribution is extremely important in interpreting the data since estimates of accuracy and directional bias must always be made in terms of the underlying response distribution. 5

\section{Discussion}

It seems fairly clear that constituent structure is not playing the critical role in the perception of clicks that has been hypothesized for it. Of the linguistic factors manipulated in this study, intonation is the most powerful in the control it exerts over the patterns of errors Ss make. Syntax is important but its effects are weak compared with intonation, and the semantic factor is of no consequence. Further, the location of the click in the word string produces large effects. At a later point, we shall present what we feel is an acceptable compromise interpretation of these results.

Intially, however, there are certain procedural issues that need to be clarified. One objection that may be made to this study is that the experimental task was so overwhelming that the Ss never were able

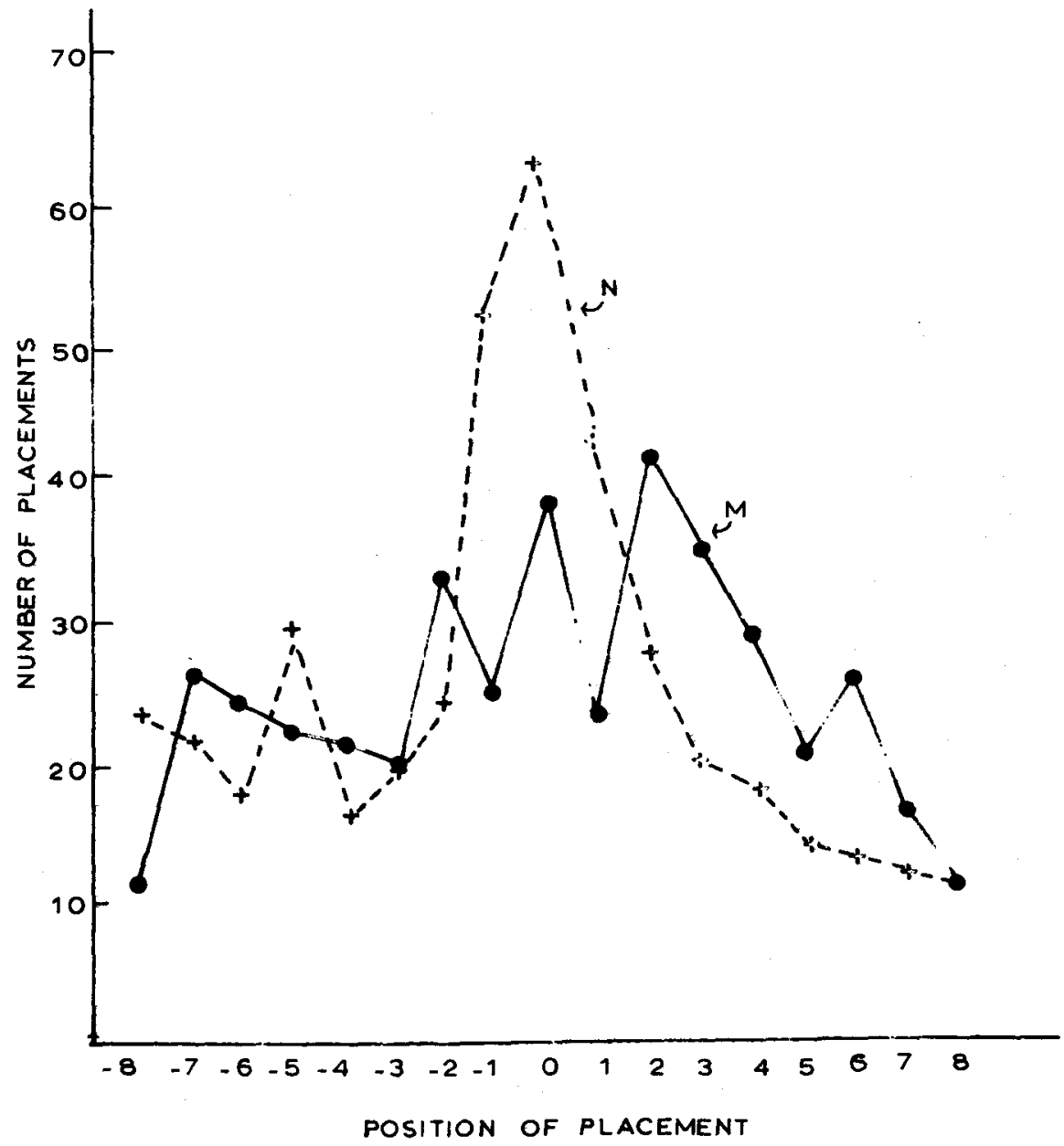


Table 3

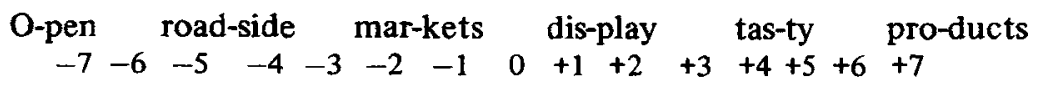

to concentrate upon the linguistic aspects of the items. Since on at least one-half of the trials the items were not even grammatical, it may be argued that $S$ s merely "gave up" listening to them as English sentences and, instead, concentrated on a more salient variable such as the intonation pattern. To try to take into account this objection, we ran the following experiment.

\section{EXPERIMENT 2}

In this study we tried to get a better understanding of the patterns of responses that occur under the most normal of conditions, NGS.

\section{Method}

Two sets of normally intonated, grammatical, semantically coherent, six-word sentences were made up. One set was composed of the same four sentences used repeatedly, in fixed order; the other set used a total of 30 different sentences. We were also interested here in whether or not the pattern of responses that Ss make would be different when they were highly familiar with and could predict the exact sentence they would hear on any given trial.

Clicks were introduced in a much more comprehensive manner in this experiment. All 15 positions from -7 to +7 , including word gaps, were used. See, for example, Table 3.

All 15 click positions were represented in four blocks of 15 trials each, with order randomized within each block. These full sets of 60 sentences were rerun three times, yielding a total of 180 judgments for each S, 12 judgments on each of the 15 click positions. As before, the stimuli were presented over stereo speakers in a quiet room, with the sentences presented over one speaker and the click over the other. Click intensity was adjusted to equal approximately that of the loudest part of the linguistic message.

There were $40 \mathrm{Ss}$; all were University of British Columbia undergraduate volunteers. They were originally divided into two groups of $20 \mathrm{Ss}$ each. One group heard the same four sentences repeated as a cycle a total of 45 times, the other group heard a cycle of 30 different sentences repeated 6 times. Two master tapes were made, using different sentences in each case to protect against any possible effects of the particular sentences used. No reliable differences were found either due to the particular sentences used or to the familiarity factor. Thus, in all the analyses to follow, the Ss were treated as one group of $40 \mathrm{Ss}$.

\section{Results}

The data again were analyzed according to the two basic measures, probability of an error $\left(\mathbf{P}_{\mathrm{e}}\right)$ and probability of an error to the right $\left(\mathbf{P}_{\mathrm{e}, \mathrm{r}}\right)$.

Probability of an error, $P_{e}$. The average $P_{e}$ was .609 , as compared with .419 from the previous experiment. This difference is probably due to a number of factors: the lack of monotone intonation that produced a much lower error rate, the increased number of click positions, and the rather boring nature of the task, to mention but a few. In any event, the aspects of the data of most concern are the pattern and direction of the errors, not the number.

An analysis of variance showed two major effects, click position, $\mathrm{F}(14,504)=8.35, \mathrm{p}<.001$, and trial blocks, $F(11,396)=2.12, \quad \mathrm{p}<.02$. The trial-block effect is largely one of a slight but significant increase $(\mathrm{p}<.01)$ in the accuracy with which $S$ s can locate the click. The click position factor (Fig. 4a) has, as before, a strong effect on $\mathrm{Ss}^{\prime}$ accuracy. The drop in $P_{e}$ with later clicks is not so marked as it was in the previous study, although average $P_{e}$ for the first seven click positions is significantly higher $(p<.01)$ than for the last seven $(.661$ and .593 , respectively). Again, when $P_{e}$ is weighted by the number of responses $\left(\mathbf{P}_{\mathrm{e}}^{\prime}\right)$, the low error rate in Position 0 disappears. $\mathrm{P}_{e}^{\prime}$ for the positions left-of- 0,0 , and those right-of 0 were $.482, .475$, and .421 , respectively. Thus, in general, these results are quite comparable with those obtained in Experiment 1.

Probability of an error to the right, $P_{e, r}$. Analysis of variance showed the same two factors to be significant with $P_{e, r}$ as with $P_{e}$ : click position, $F(14,504)=8.29$, $\mathrm{p}<.001$, and trial blocks, $F(11,396)=2.48, p<.01$. The trial block effect, as in Experiment 1 , is essentially a shift from an initial left-bias on early trials to a right-bias on later trials. The overall bias in the experiment was to the left, with mean $P_{e, r}$ of .482 . This average bias is due to a strong initial left-bias $\left(\mathrm{P}_{\mathrm{e}, \mathrm{r}}=.38, .39\right.$ on the first two trial blocks) and an asymptotic $P_{e, r}$ of approximately .5 , a value somewhat below that found in Experiment 1.
The effect of click position on $P_{e_{1}}$ is shown in Fig. $4 \mathrm{~b}$. The curve is similar in most respects to the average curve from Experiment 1 (Fig. 2a). The decrease in $P_{e, r}$ across click positions is evident and reliable $(\mathrm{p}<.001)$, and clicks in Positions $-1,0$, and +1 show the same general pattern. The tendency to move Position -1 clicks to the right is greater than the tendency to move Position +1 clicks to the left, and the majority of Position 0 errors are to the right.

As before, an analysis of the error data simply in terms of constituent structure shows agreement with the Fodor and Bever hypothesis, with .654 of the errors either toward or into the major syntactic break. This value agrees nicely with that for the NGS items in Experiment 1 and with the value Fodor and Bever report.

\section{Discussion}

Two broad aspects of the data from these two experiments are important. First, the fact that we consistently obtained agreement with Fodor and Bever when we performed data analyses similar to theirs argues that we have not distorted the paradigm by introducing our stringent experimental controls. Second, the fact that deeper analyses of the data revealed unexpected results seriously challenges their interpretation. The clear implication is that the data from their study and others (Garrett, 1965; Garrett, Bever, \& Fodor, 1965; Bever, Lackner, \& Stolz, 1969; Bever, Lackner, \& Kirk, 1969) are in large part determined by factors that have not
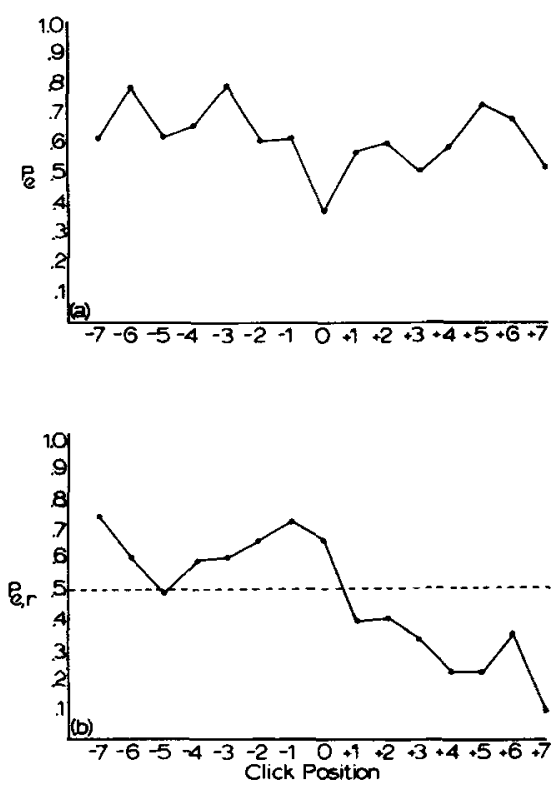

Fig. 4. (a) $P_{e}$ and (b) $P_{e, r}$ by click position for the sentences in Experiment 2. 
Table 4

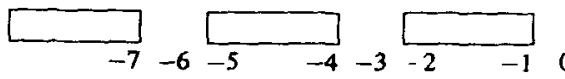

0

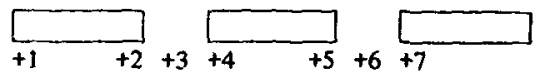

been systematically analyzed.6,7 The following effects from the two experiments were all highly reliable and present a considerable interpretive challenge: (1) Certain click positions produced a higher error rate than others, with later-occurring clicks being easier to locate; (2) there was no evidence that clicks occurring at the major syntactic break were easier to locate than clicks occurring elsewhere; (3) early trials revealed an overall left-bias in click placement which disappeared on later trials; (4) clicks occurring early in an item produced a right-bias in location, clicks occurring toward the end of an item produced a left-bias; and finally, (5) normal intonation produced a different pattern of errors than did monotone intonation.

We feel that the following tentative conclusions are justified at this point: (1) Of the various linguistic factors evaluated, intonation is clearly the most significant in terms of the control it exerts over Ss' response patterns, while syntax is relatively unimportant and semantics is irrelevant. (2) Much of the data is probably due to other nonlinguistic factors, such as attention, memory, and response biases.

The following study was designed to try to evaluate the role of these nonlinguistic factors.

\section{EXPERIMENT 3}

This final experiment was designed to be a replication of Experiment 2 except that in place of the six-word grammatical sentence the stimulus items consisted of white noise. This technique allows us to determine the extent to which the data from the previous studies are independent of linguistic factors.

\section{Method}

The stimulus items were sequences of six bursts of white noise $(0-10,000 \mathrm{~Hz}$, uniform distribution). Each burst had a duration of $370 \mathrm{msec}$, with a rise and fall time of $10 \mathrm{msec}$. The interval between noise bursts was $110 \mathrm{msec}$. Thus, a full stimulus item had a duration of $2.77 \mathrm{sec}$, a value that approximated the average duration of the normally intonated, grammatical sentences used in the previous studies.

The click again was a spot of broad-band square-wave noise with a physical duration of $.05 \mathrm{msec}$. Its intensity was set equal to that of the noise bursts. A total of 15 click positions was used; they corresponded to those used in Experiment 2, e.g., see Table 4.

The clicks corresponding to those in the word gaps $(-6,-3,0,+3,+6)$ were placed in the center of the silent interval, clicks corresponding to those over the first syllable $(-5,-2,+1,+4,+7)$ were placed $90 \mathrm{msec}$ from the beginning of the rise of the noise burst, those over the second $(-7$, $-4,-1,+2,+5)$ were placed $90 \mathrm{msec}$ from the end of the fall of the noise burst.

The placement of clicks was randomized within blocks of 15 trials so that each block contained one representation of each. Two separate master tapes consisting of four of these randomized blocks were made up. For each experimental session, one of the tapes was rerun three times so that each $S$ judged a total of 180 click placements. There was no difference between the two randomizations, and analyses were carried out on a pooled sample.

As in the previous experiments, response booklets were prepared for Ss. After hearing a complete stimulus, $S$ turned the blank divider page and indicated his response by placing an arrow over the position that he perceived the click. As in Experiment 2, Ss were instructed that all response alternatives, including those outside the noise burst train, were permissible. To eliminate any possible ambiguity about the placement of a particular click, the response sheets were arranged as in Table 5. All stimulus materials were placed directly on the tape electronically. The experimental sessions were conducted in a quiet room free from distraction. The tapes were played on a stereo tape recorder with the noise burst train on one channel and the clicks on the other. The Ss were 20 undergraduates at the University of British Columbia.

\section{Results}

There was a total of 3,600 responses; they were analyzed according to the same two measures, $P_{\mathrm{e}}$ and $\mathbf{P}_{\mathrm{e}, \mathrm{r}}$ :

Probability of an error, $P_{e}$. The average probability of an error was .521 , a value about half-way between that observed in the two previous experiments. An analysis of variance revealed only one significant factor, click position, $F(14,252)=1.82$, $p<.05$. Figure 5a shows that $P_{e}$ decreases systematically with late click positions, with Position 0 showing the lowest error rate. Since this study contains no sentential material, it is now clear that Ss' response biases are the major factor contributing to the low $P_{e}$ at Position 0 and memory is the major factor contributing to the reliable linear decrease $(p<.05)$ in $P_{e}$ for late-occurring clicks. When $P_{e}$ is corrected for total number of responses $\left(P^{\prime}\right)$ the values for clicks left-of- 0,0 , and right-of-0 are $.507, .527$, and .472 , respectively

Probability of an error to the right, $P_{e, r}$. The average probability of an error to the right was .527. An analysis of variance showed that only click position had a significant effect on the direction of an error, $F(14,252)=1.88, p<.05$. As in the previous studies, clicks occurring to the left of center produced errors to the right and those to the right generally produced errors to the left. The cross-over point where the right-bias shifts to a left-bias is even a bit further to the right here than in Experiments 1 and 2.

The failure to find a trial block effect in this study, $F(11,198)=.93$, is intriguing. In both of our previous studies, as well as in the experiments reported by others (Ladefoged \& Broadbent, 1960; Fodor \& Bever, 1965), an initial left-bias was found that gradually gave way to a right-bias. Here, however, the very first block of trials produced a $P_{e, r}$ of .501. This experiment
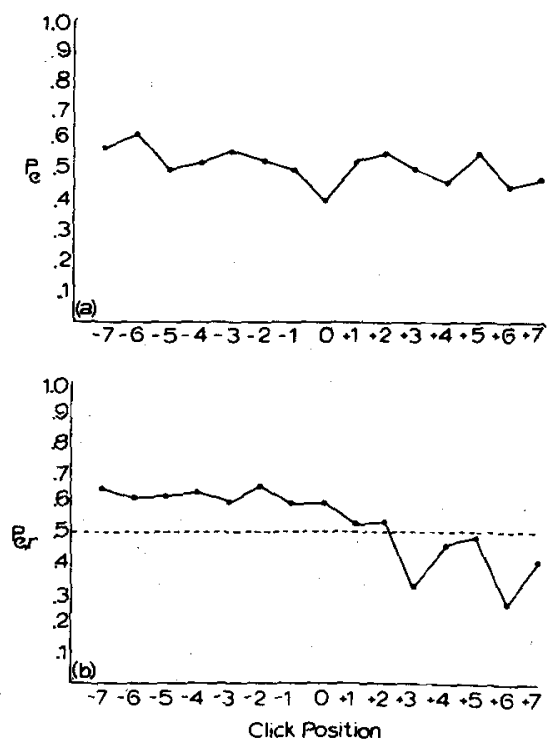

Fig. 5. (a) $P_{e}$ and (b) $P_{e, r}$ by click position for the noise bursts in Experiment 3. 


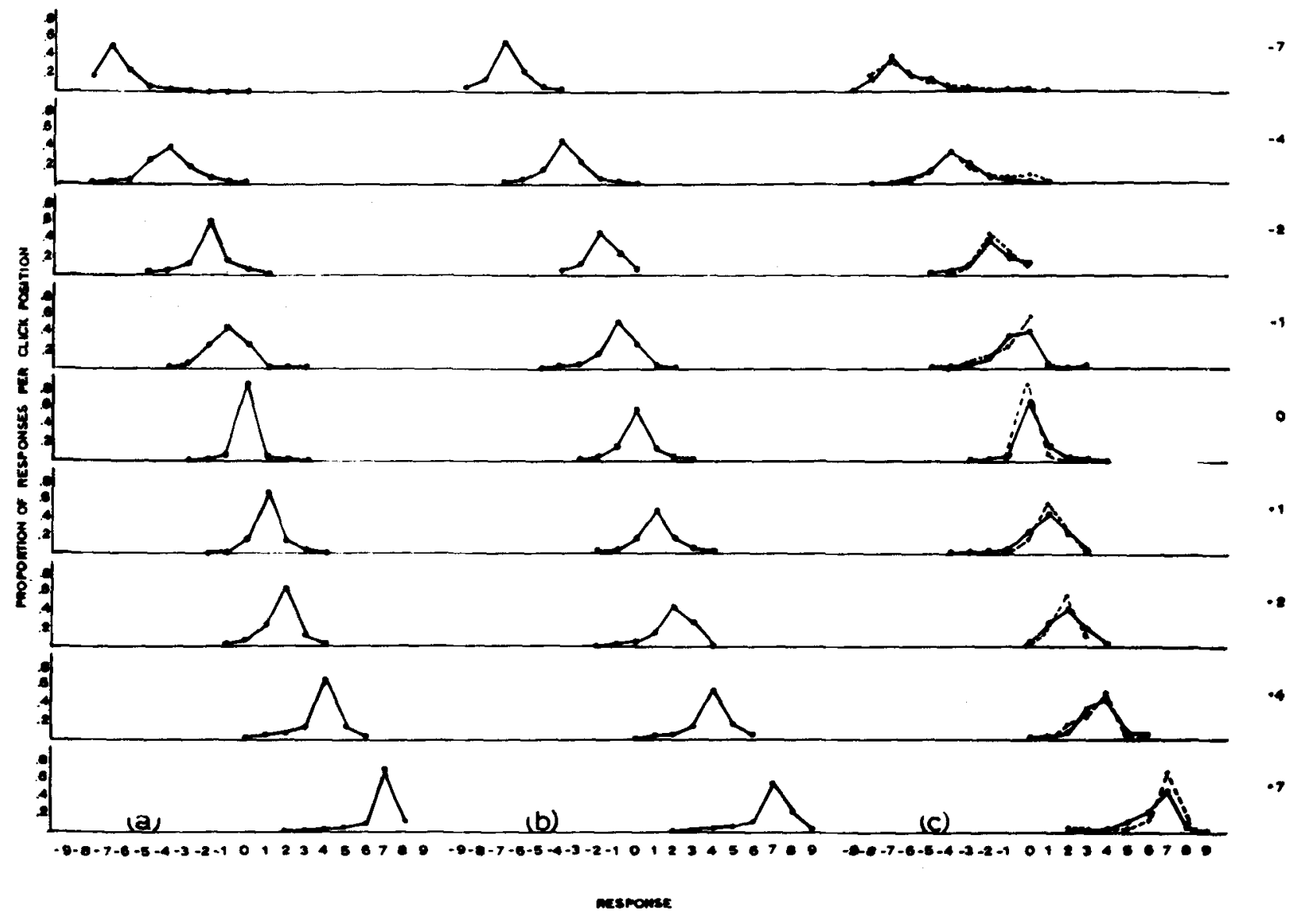

Fig. 6. Full confusion matrices showing the distribution of responses made by all Ss to each click position in (a) Experiment 1 , all conditions pooled, (b) Experiment 3, and (c) Experiment 2 (solid lines) and the NGS items from Experiment 1 (dotted lines).

differs from the others primarily in that the stimuli were nonlinguistic. If, as we shall argue below, the directional bias is a result of Ss' attentional processes, then clearly the noise bursts are attended to, at least initially, in a different manner than sentential material.

Once more, there is superficial evidence that Ss are misperceiving clicks so that they appear to be "attracted" by Position 0 . Of all the errors in the experiment, $56.7 \%$ of them were toward Position 0. However, in this experiment there is no syntactic boundary that can be attracting the clicks.

\section{GENERAL DISCUSSION}

The data from these three studies are more striking for the similarities among them than for the differences between them. The same general pattern of errors was found regardless of whether the stimulus items were English sentences or bursts of white noise. These similarities are most easily seen by looking at the full confusion matrices in Figs. 6 and 7, where all the data from each experiment is presented in its most compact form. These figures show the frequency distributions of all responses made by all $\mathrm{Ss}$ to each of the click positions. Figure 6 shows (1) the pooled confusion matrices from all conditions in Experiment 1, (2) the matrices from the noise burst study, and (3) the matrices from the six-word sentences from Experiment 2 (solid lines) and the matrices from the NGS condition from Experiment 1 (dotted lines). Note that Click Positions $-6,-5,-3,+3,+5,+6$ are not included in (2) and (3) so that direct comparisons with the data in (1) can be carried out. Figure 7 shows the full confusion matrices from Experiments 2 and 3 , including the click positions omitted from Fig. 6.

Kolmogorov-Smirnov tests were run on all interexperiment comparisons within each click position. There were no significant differences. Clearly, then, an interpretation of the click migration phenomenon must be based, in the final analysis, upon nonlinguistic factors.

Most of the data from these studies can now be distilled down to three generalizations: (1) Clicks located early in an item are perceived as occurring later than their actual placement; (2) clicks occurring late in an item are perceived as occurring earlier than their actual placement; and (3) these two directional biases are not necessarily symmetric around the central position.

We suggested briefly that nonlinguistic mechanisms, particularly those of attention and memory, were important in these experiments. The following hypothesis represents a preliminary effort to formalize some of these suggestions.

\section{A Neo-Titchenerian Hypothesis}

Three assumptions are needed: (1) The perceptual process is fundamentally a single-channel operation with only one channel attended to at any one time; (2) Ss will initially attend to the channel containing the most novel auditory input; and (3) inputs in the attended channel are perceived as occurring earlier in time than are contiguous inputs on the nonattended channel.

None of these assumptions is original. The first is merely a simplification of the 

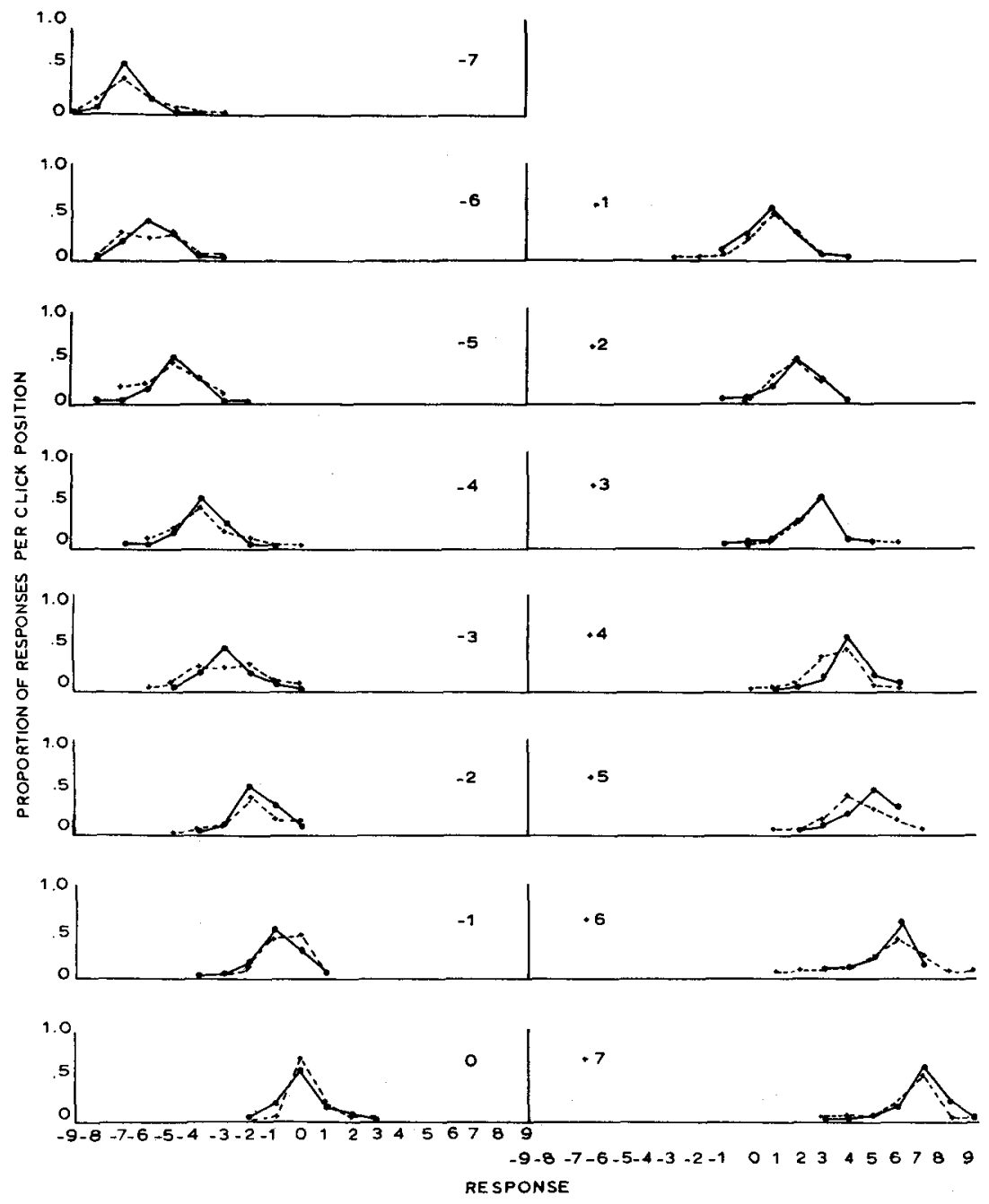

primary axiom of Broadbent's (1958) theory of perception. The second is supported by the research of Berlyne (1950, 1957), Poulton (1965), and Cantor and Cantor (1966), among others, who have shown that with rats, adults, and children the more novel stimulus is attended to sooner. The third is merely a restatement of Titchener's (1909) law of prior entry.

The behavior of our Ss can thus be characterized as follows:

(1) During the initial phases of the experiments with sentential material, the click constitutes a more unusual and novel acoustic stimulus than the linguistic input, and Ss will attend to the channel containing the click rather than to the one containing the human voice. Thus, initial trials will display an overall left-bias. On the later, asymptotic trials, after Ss have familiarized themselves with the clicks, attention is distributed equally between the two inputs, and the average bias is effectively at a chance level. The failure to
Fig. 7. Full confusion matrices showing the distribution of responses made by all Ss to each click position in the grammatical sentences of Experiment 2 (dotted lines) and the noise bursts of Experiment 3 (solid lines).

for it. Second, as the item progresses it becomes more and more determined and redundant and $S s$ can safely redirect their attention. The fact that normal intonation produced a very large shift in $P_{e, r}$ with click positions in Experiment 1, as compared with monotone intonation, is interesting since normal intonation produces very marked cues to the $S$, indicating progress through the item. It may be argued, then, that the primary role of the linguistic factors is one of controlling the manner in which Ss partial out their single perceptual channel. Note, for example, that the decrease in $P_{e, r}$ in Experiment 2 was considerably more marked than it was in Experiment 3. When noise bursts are the primary message the linguistic redundancy is missing, and somewhat more progress must be made through the item before Ss switch their attention. Hence the cross-over from rightto left-bias in Experiment 3 doesn't occur until roughly Position +2 .

From the foregoing, it appears that this attentional interpretation can account for a considerable portion of the data with relative ease. There are some problems and they have implications for future research. First, all the verbal stimulus items used in the experiments were symmetric with the major syntactic boundary located in the central position. This fact is not critical since the data were asymmetrical, but it is possible that varying the position of the major boundary might affect Ss' attentional priorities.

Second, there is no provision in the neo-Titchenerian hypothesis for the differential error rates found at the different click positions. It is probably the case that low $P_{e}$ at Position 0 is due to a response bias and that low $P_{e}$ at late click position is due to memory factors. Nevertheless, it would be desirable to develop a technique for eliminating Ss' response biases so that error rates can be explored unambiguously.

Third, the monotone intonation pattern in Experiment 1 produced data that would agree only weakly with this interpretation. An $M$ intonation pattern, however, yields items that are approximately $5 \frac{1 / 2}{\mathrm{sec}}$ in duration while all others averaged only about $3 \mathrm{sec}$. We do not know what the effects on attention might be of different item durations.

Fourth, it is quite possible that a variety of basic psychophysical mechanisms are 
operating in these experiments. For example, Ss may be locating click positions by estimating the time between the occurrence of the click and one of the anchor points - the beginning and end of an item. There is evidence that under such conditions (M. Humphreys ${ }^{8}$ ) Ss tend to display asymmetrical estimates.

In summary, it seems safe to conclude that the interpretations of the click migration phenomena based upon linguistic decoding operations are an oversimplification. We do not wish to imply that such factors as constituent structure are not relevant in speech perception, merely that the patterns of errors that Ss make in attempting to locate clicks in linguistic messages should not be regarded as unambiguous support for the use of these units.

\section{REFERENCES}

BERLYNE, D. E. Novelty and curiosity as determinants of exploratory behavior. British Journal of Psychology, 1950, 41, 68-80.

BERLYNE, D. E. Attention to change. British Journal of Psychology, 1951, 42, 269-278.

BEVER, T. G., LACKNER, J. R., \& KIRK, R. The underlying structures of sentences are the primary units of immediate speech processing. Perception \& Psychophysics, 1969, 5 225-233.

BEVER, T. G., LACKNER, J. R., \& STOLZ, W. Transitional probability is not a general mechanism for the segmentation of speech Joumal of Experimental Psychology, 1969, 79, 387-394.

BROADBENT, D. E. Perception and communication. London: Pergamon Press, 1958.

CANTOR, G. N., \& CANTOR, I. H. Discrimination reaction time in children as related to amount of stimulus familiarization. Joumal of Experimental Child Psychology, $1966,4,150-157$.
FELDMAR, A. The perception of clicks embedded in sentences: Encoding or decoding? Unpublished Master's thesis, University of Western Ontario, 1969.

FODOR, J. A., \& BEVER, T. G. The psychological reality of linguistic segments. Journal of Verbal Learning \& Verbal Behavior, $1965,4,414-420$.

GARRETT, M. Syntactic structures and judgements of auditory events. Unpublished doctoral dissertation, University of Illinois, 1965.

GARRETT, M., BEVER, T. G., \& FODOR, J. A. The active use of grammar in speech perception. Perception \& Psychophysics, 1966, 1, 30-32.

LADEFOGED, P. Three areas of experimental phonetics London: Oxford University Press, 1967. Chap. 3.

LADEFOGED, P., \& BROADBENT, D. E. Perception of sequence in auditory events. Quarterly Journal of Experimental Psychology, 1960, 12, 162-170.

MARKS, L. E., \& MILLER, G. A. The role of semantic and syntactic constraints in the memorization of English sentences. Journal of Verbal Learning \& Verbal Behavior, 1964, 3 , $1-5$.

MILLER, G. A., \& ISARD, S. Some perceptual consequences of linguistic rules. Journal of Verbal Learning \& Verbal Behavior, 1963, 2, 217-228.

NEISSER, U. Cognitive psychology. New York: Appleton-Century-Crofts, 1967.

POULTON, E. C. Listening to overlapping calls. Journal of Experimental Psychology, 1956, $52,334-339$.

TITCHENER, E. B. Lectures on the psychology of the thought processes. New York: Macmillan, 1909.

1. Supported in part by a grant from the University of British Columbia Committee on Research. Experiment 1 is based upon the Honours Thesis of J. R. Anderson.

2. Address: Department of Psychology, University of British Columbia, Vancouver, B.C., Canada.

3. Now at Stanford University.

4. X.X. Kaplan, personal communication.

5. This notion of the Ss' response bias, while critical for interpreting click location data, is itself a particularly difficult one to understand. The subliminal control showed cleariy that Ss tended to think they heard clicks in the cente position more frequently than in any other position. This tendency could be "perceptual" or it could be due simply to a nonuniform confidence component. That is, for some reason, Ss may be reluctant to select a position outside of Position 0 unless they are quite confident that they are correct. Thus the Position 0 data may be distorted by a high false-alarm rate. Unfortunately, we did not collect confidence ratings from our $\mathrm{Ss}$ and cannot test this interpretation directly.

6. It should be pointed out here that there are at least two methodological problems in the Bever, Lackner, and Stolz study. First, there was no control of the syllabic structure of the words over which the clicks were placed. It is not clear that errors in locating a click over the word "musician" and "eat" represent the same process. If clicks were centrally placed, the former is an error of three positions and the latter an error of only one position. Second, there was no control of the placement of clicks relative to the center of sentences. The average click, in fact, was .33 words to the left of center, a position that we argue should produce a marked right-bias. However, all the experimental effects that Bever et al report involved a tendency to locate clicks to the right of the actual position. In view of our results, it is not clear that this effect can be unambiguously interpreted. A control experiment involving a replication with click positions balanced around the center is called for

7. It is not clear from the results of the Bever, Lackner, and Kirk study whether the different distributions of errors in the various linguistic conditions are due to a perceptual process under the control of underlying structural factors or merely due to different response biases. A recent study by Feldmar (1969) showed that the Ss' response biases shift with shifts in syntactic structure. The subliminal control is absolutely critical in studies of this nature and without it meaningful conclusions cannot be drawn.

8. M. Humphreys, personal communication.

(Accepted for publication October 27, 1969.) 